\title{
ANALYSIS OF EMOTIONS USING EEG DATA AND MACHINE LEARNING
}

\author{
Vyacheslav V. Potekhin \& Ogul Unal
}

Peter the Great St. Petersburg Polytechnic University
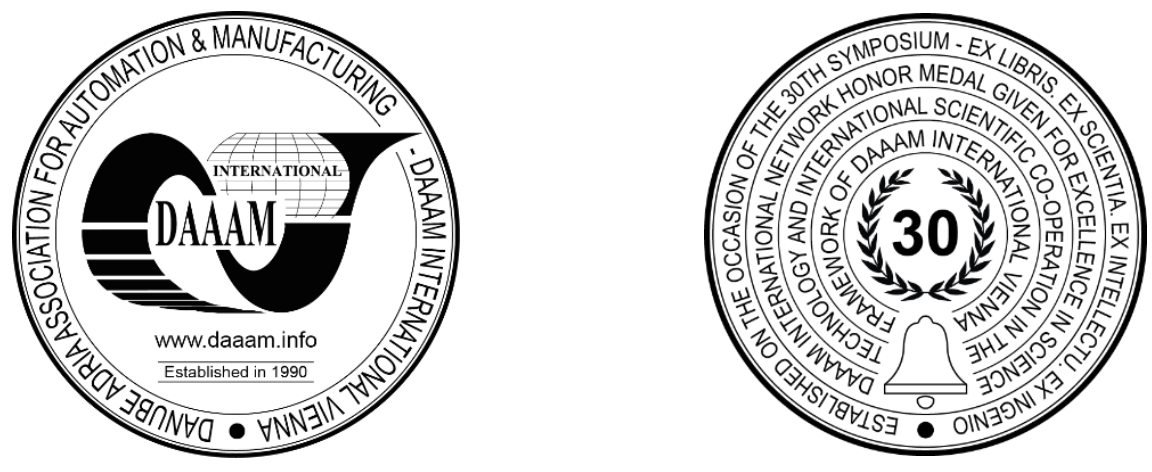

This Publication has to be referred as: Potekhin, V[yacheslav] \& Unal, O[gul] (2021). Analysis of Emotions using EEG Data and Machine Learning, Proceedings of the 32nd DAAAM International Symposium, pp.0158-0167, B. Katalinic (Ed.), Published by DAAAM International, ISBN 978-3-902734-33-4, ISSN 1726-9679, Vienna, Austria DOI: $10.2507 / 32$ nd.daaam.proceedings.025

\begin{abstract}
Emotions might be one of the most significant differences between a machine and a human being. Currently, Machine Learning can learn and make predictions using Data in many different fields such as Medicine. In this paper, the goal is to evaluate how human emotions are being executed then test the performance of ML in terms of Emotion analysis. DREAMER Data was used to perform all the tasks. The classification of rankings for 23 subjects' was done. The reactions of subjects and EEG data were recorded while 23 participants were watching 18 different short movies. Each candidate has a ranking, between one to five, based on their affective state after each stimuli in terms of valence, arousal, and dominance. Afterward, Using Generative Adversarial Network (GAN), synthetic data will be created and analysed using supervised and unsupervised learning algorithms. Finally, all results will be compared. The research can help to evaluate the basic human emotions for robots or devices in Medicine. Robotics or cyber-physical machines in healthcare are already growing every day therefore, the quality of surgeries, treatments, or medical assistance can be improved.
\end{abstract}

Keywords: Machine Learning; Emotion Analysis; Electroencephalography; Brain Computer Interface; Generative Adversarial Networks

\section{Introduction}

Electroencephalography, according to the American Heritage dictionary, is a neurological test that works with electronic monitoring equipment to estimate and document the electrical activity in the brain. EEG can monitor and record the electrical activity of the human brain. Therefore it is possible to use a Medicine Dataset for Machine Learning (ML) algorithms. Today, many kinds of research are aiming to solve how the human brain controls our emotions and actions. Several examples are; Facial expression recognition or Emotion analysis using a Human-machine interface.

Today, the human brain is not fully understood. It is likely to get the unique language occurring in the human brain with the help of signal processing technology and instruments and transform them into machine understandable language. Therefore in this project, it was possible to use EEG Affect Recognition Dataset (DREAMER) based on the signals. Emotion theories and brain structure have been studied. Afterward, neurons and neurotransmitters were analysed to understand the electrochemical structure of the brain. 
The Supervised, Unsupervised, and Generative Adversarial Networks in the Machine Learning field have been used to perform experiments to understand the dataset in detail. The classification tests had significantly high accuracy results therefore, it is possible to use the Machine Learning approach in affect recognition systems. The results might be useful to provide more information to new technical solutions related to human emotions in intelligent systems.

Due to the importance of the health industry in the world and the overpopulation, robots might help in the healthcare systems thus, intelligent machines can assist people. This research aims to study human emotions that are unknown and difficult to understand mathematically and explain emotions to computers using mathematics, EEG, and machine learning. Brain structure is not fully understood. It is aimed that this research will contribute to the development of the connection between the human brain and computers and help patients in hospitals.

\section{Literature Review}

\subsection{The Human Brain}

Approximately one and a half kilogram organ that organizes every function to manage the body itself. The brain is also responsible to analyse the information as well as controlling emotions, intelligence, and memory. The main parts of the brain are Cerebrum, Cerebellum, and Brainstem [1]. The five senses can receive by the brain as tasting, touching, hearing, smelling, and sight simultaneously. It can assemble any message that is meaningful for us because the brain can collect and interpret the memory. Instincts, desires, pain happiness, and any other emotional condition is controlled by the brain.

\subsection{Emotions in Human Brain}

The limbic system consists of parts such as the thalamus, hypothalamus, hippocampus, and amygdala. The limbic system is also called the emotional brain because it is the control center for emotions. The limbic system is associated with emotions [7]. Thus, the limbic system governs behaviours, exciting events, and behaviours necessary for the survival of the individual. The other parts of the brain and the limbic system work together to regulate purposeful behaviours according to the information coming from the inner and outer environment of the person.
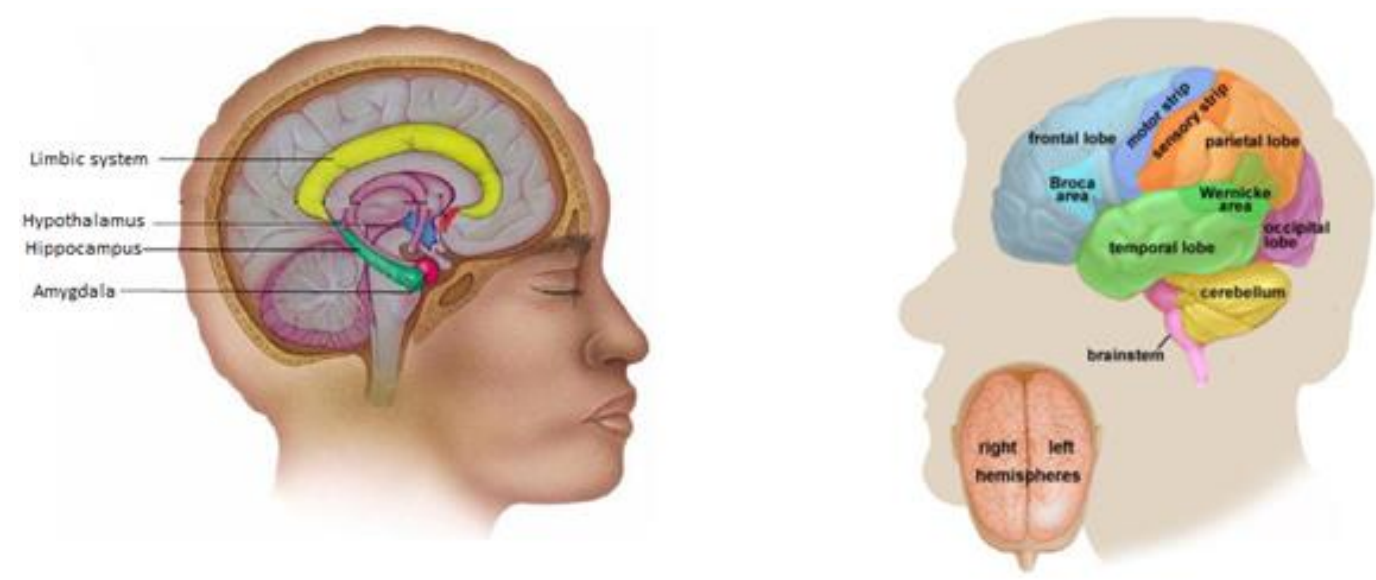

Fig. 1. Human brain structure (left) and the Limbic System (right)

Source: www.macalester.edu/psychology

\subsection{Neurons and Nervous System}

The cells that make up the nervous system are called neurons. The neuron consists of three main parts. Those are the cell body (soma), dendrite, and axon. Dendrites are numerous and usually short extensions [7]. These extensions receive stimuli from receptors and other nerves. The action potential is the brief electrical event that is generated in the axon which signals the neuron as "active". An action potential travels as the length of the axon and causes the release of neurotransmitters into the synapse. The action potential and consequent transmitter release allow the neuron to contact other neurons. The nervous system is the organized group of cells specialized for the conduction of electrochemical stimuli from sensory receptors through a network to the site at which a response occurs. [18].

Electrophysiology of a neuron; The general idea of electrical signals in a neuron is; there is a voltage difference between the interior of the neuron relative to the space surrounding the neuron, the extracellular space. This voltage is known as the membrane potential which is a constant of about $-70 \mathrm{mV}$ [8]. When a neuron is stimulated, the membrane voltage may indicate both positive and negative variants. These voltage variations could carry signals across the body of a neuron, and also contribute to signals that are broadcasted from one to another across the synapse [8]. 


\subsection{Neurotransmitters}

Neurons are communicating through the body and they communicate with one another to transmit signals. Although, neurons are not simply connected physically. Each neuron's end is a small gap that is a synapse and to communicate with the next cell, the signal has to be able to cross the space. This process is known as neurotransmission. There are excitatory and inhibitory neurotransmitters. Excitatory will increase the likelihood that the neuron will fire an action potential while inhibitory will decrease it. The functions of the neurotransmitters are explained and shown below.

Neurotransmitters and their functions are explained as [6]:

- Adrenaline (excitatory): Also called Epinephrine, it increases blood flow and heart rate.

- Norepinephrine: (NE): also known as noradrenaline (excitatory) Increases blood flow and attention.

- Dopamine (both excitatory and inhibitory): is mostly responsible for feelings of reward and pleasure. Abnormal dopamine level is common in specific disorders such as schizophrenia [2].

- Serotonin (inhibitory): feelings of happiness and well-being, stable mood, sleep cycle, and digestive system.

- Gamma-aminobutyric acid (GABA) (inhibitory): Inhibits neuron firing in the Central Nervous System also, high levels improve focus and low levels causes anxiety, contributes to motor control and vision.

- Acetylcholine(excitatory): learning, memory, muscle contraction, awakening

- Glutamate (excitatory): learning, memory, and creating new nerve pathways.

- Endorphins: natural pain killer, excitement, and exercise.

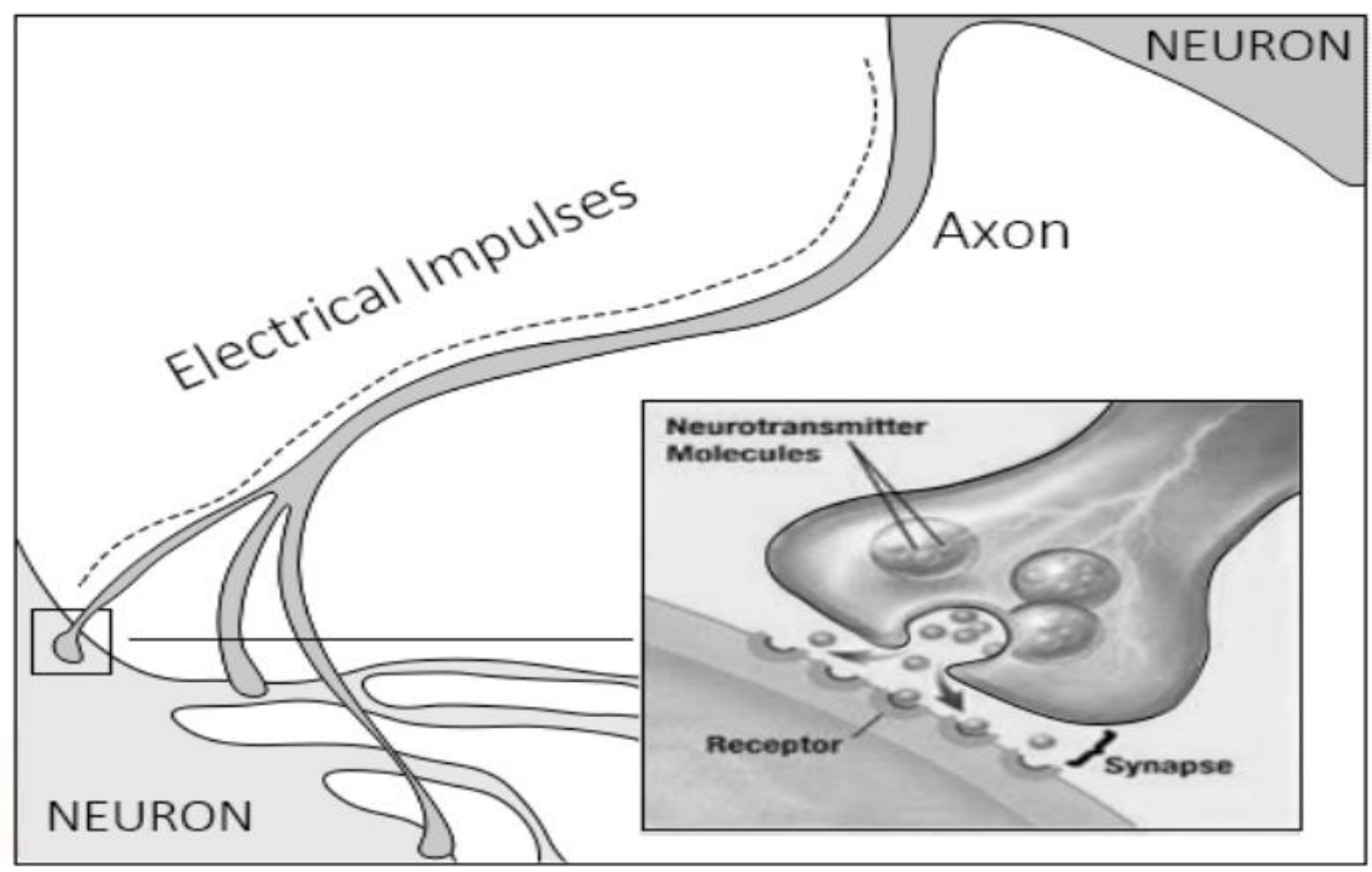

Fig. 2. Illustration of the major elements in chemical synaptic transmission

\subsection{Emotions and Neurotransmitters}

Neurotransmitters and have different physiological signatures while transmitting signals throughout the brain. Currently, there are many unknown areas about complex emotions in the human brain, basic emotions might be detected in the responsible areas [10]. Basic emotions such as fear, anger, pleasure (when combined) create another layer for creating different types of secondary emotions, therefore, primary emotions can be combined with the experience of other, higher-order emotions that are more affected by specific learning and experience [11]. Using this approach, primary emotions are observable in evolutionarily diverse organisms, which allows us to functionally "dissect" the mechanisms of the presumed associated internal emotional states and their externally manifest behaviours [11]. The first important theory about emotions is Basic Emotion Theory which is evolved to handle fundamental life tasks, e.g., fear and anger can aid survival. The second theory is the Dimensional Theory of Emotion that has three types of emotions those are: pleasant-unpleasant, tension-relaxation, and excitation-calm [11]. 

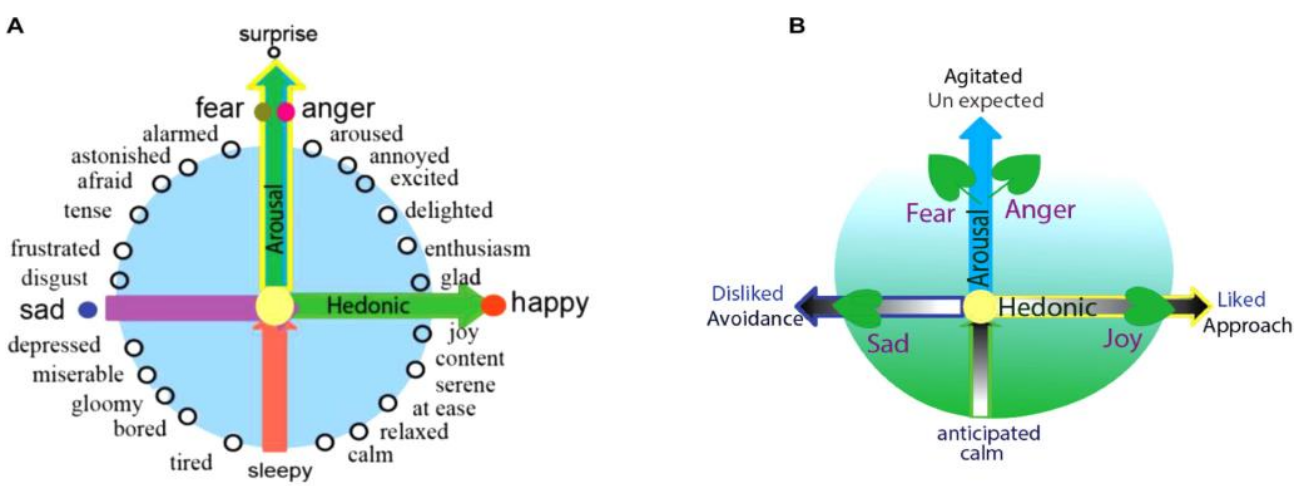

\section{$2.6 E E G$}

Fig. 3. Dimensional Theory of Emotion

The brain has hundreds of billions of neurons that are performing electrical transmissions with densely interconnected via of the synapses. The nerve cells in our brains are active they create electrical signals that rotate through the surface of the scalp. Thus by using sensors placed on the scalp it is viable to measure those changes in Voltage while performing an activity or solving a task [3]. The measurement of EEG divides into two parts that are considered as Non-Invasive and Invasive techniques. In this research, all tasks were under Non-Invasive systems.

It is possible to say that the source of EEG signals is the pyramidal cells in the cortex. All cells in the corresponding area are perpendicular and parallel to each other. Each pyramidal cell has approximately 30000 exhibitory and 1700 inhibitory inputs [4]. Thus the EEG is created by the electrical flow of neurons that are placed in extracellular space. The parallel configuration is; the Pyramidal neurons are spatially aligned perpendicular to the articular surface. Thus it creates a dipole layer in the cortex. Therefore EEG represents the mainly postsynaptic potentials of those pyramidal neurons which are close to the recording electrode [5].

\section{Methods \& Materials}

\subsection{EEG and DREAMER Database}

EEG nodes, Participant number, and Age columns were used in the dataset for Machine Learning. Gender 0 is Male, Gender 1 represents Female subjects. It is not fully understood differences of emotions for different genders therefore in this research, Genders have been separated and tested individually. From EEG nodes near to limbic to were used.

DREAMER is a multi-modal database containing electroencephalogram (EEG) and electrocardiogram (ECG) signals recorded. Signals from 23 participants were recorded along with the participants' self-assessment of their affective state after each stimulus, in terms of dominance, valence, and arousal. All the signals were captured using wearable, portable and wireless equipment. [20]. The Emotiv-EPOC wireless EEG headset was used for EEG The database contains the participant ratings and physiological recordings of an experiment where 23 volunteers have watched 18 film clips [19]. EEG and ECG signals were recorded and each participant rated their emotion by reporting the felt arousal, valence, and dominance on five-point scales. The peak of the main wave of AI is about to hit industries, governments, and societies accelerating the pace of product and service innovation and putting data in the focus of the business interest [9]. Using $\mathrm{AI}$ in Medicine is also another challenge nowadays. Advanced distributed coordination and decision-making techniques allow more effective operation and availability therefore it is possible to combine AI, Medicine, and a cyber-physical system to build a human-computer interface [28].

In this work, only the EEG Stimuli part of the Database was used to perform Machine Learning experiments. Dataset was created based on 23 participants that watched 18 films. Out of 18 films, nine different film clips were chosen. Each of these nine films targets emotions as shown in the figure below. Each prepared dataset contains one film for 23 subjects. Each subject's results were appended to the next one respectively. During the ML experiment datasets were shuffled, validated and Random State was used to avoid overfitting.

\begin{tabular}{|c|c|c|c|c|c|c|c|c|c|c|c|c|c|c|c|}
\hline & & F7 & F3 & FC5 & $\pi$ & T8 & FC6 & F4 & F8 & Participant & Gender & Age & ScoreValence & ScoreArousal & ScoreDominance \\
\hline & 0 & 4110.769231 & 4080.000000 & 4336.923077 & 4277.948718 & 4232.820513 & 3873.846154 & 4414.871795 & 4288.205128 & 1 & 0 & 22 & 5 & 4 & 4 \\
\hline & 1 & 4101.538462 & 4106.153846 & 4392.820513 & 4341.025641 & 4309.743590 & 3919.487179 & 4425.641026 & 4334.871795 & 1 & 0 & 22 & 5 & 4 & 4 \\
\hline & 2 & 4097.948718 & 4106.666667 & 4398.974359 & 4346.153846 & 4330.256410 & 3929.230769 & 4424.615385 & 4350.256410 & 1 & 0 & 22 & 5 & 4 & 4 \\
\hline & 3 & 4110.256410 & 4089.230769 & 4356.923077 & 4299.487179 & 4272.307692 & 3896.410256 & 4420.000000 & 4319.487179 & 1 & 0 & 22 & 5 & 4 & 4 \\
\hline & 4 & 4121.538462 & 4092.820513 & 4346.153846 & 4295.897436 & 4251.282051 & 3882.051282 & 4428.205128 & 4315.384615 & 1 & 0 & 22 & 5 & 4 & 4 \\
\hline
\end{tabular}

Fig. 4. The first 5 lines of each of 9 Supervised Dataset in python 


\begin{tabular}{lccccc}
\hline \hline ID & Film clip & Target emotion & Valence & Arousal & Dominance \\
\hline 1 & Searching for Bobby Fischer & calmness & $3.17 \pm 0.72$ & $2.26 \pm 0.75$ & $2.09 \pm 0.73$ \\
2 & D.O.A. & surprise & $3.04 \pm 0.88$ & $3.00 \pm 1.00$ & $2.70 \pm 0.88$ \\
3 & The Hangover & amusement & $4.57 \pm 0.73$ & $3.83 \pm 0.83$ & $3.83 \pm 0.72$ \\
4 & The Ring & fear & $2.04 \pm 1.02$ & $4.26 \pm 0.69$ & $4.13 \pm 0.87$ \\
5 & excitement & $3.22 \pm 1.17$ & $3.70 \pm 0.70$ & $3.52 \pm 0.95$ \\
6 & disgust & $2.70 \pm 1.55$ & $3.83 \pm 0.83$ & $4.04 \pm 0.98$ \\
7 & National Lampoon's VanWilder & happiness & $4.52 \pm 0.59$ & $3.17 \pm 0.98$ & $3.57 \pm 0.99$ \\
8 & Wall-E & anger & $1.35 \pm 0.65$ & $3.96 \pm 0.77$ & $4.35 \pm 0.65$ \\
9 & Crash & sadness & $1.39 \pm 0.66$ & $3.00 \pm 1.09$ & $3.48 \pm 0.95$ \\
10 & My Girl & disgust & $2.17 \pm 1.15$ & $3.30 \pm 1.02$ & $3.61 \pm 0.89$ \\
11 & The Fly & calmness & $3.96 \pm 0.64$ & $1.96 \pm 0.82$ & $2.61 \pm 0.89$ \\
12 & Pride and Prejudice & amusement & $3.96 \pm 0.56$ & $2.61 \pm 0.89$ & $2.70 \pm 0.82$ \\
13 & Modern Times & happiness & $4.39 \pm 0.66$ & $3.70 \pm 0.97$ & $3.74 \pm 0.96$ \\
14 & Remember the Titans & anger & $2.35 \pm 0.65$ & $2.22 \pm 0.85$ & $2.39 \pm 0.72$ \\
15 & Gentlemans Agreement & fear & $2.48 \pm 0.85$ & $3.09 \pm 1.00$ & $3.22 \pm 0.9$ \\
16 & Psycho & excitement & $3.65 \pm 0.65$ & $3.35 \pm 1.07$ & $3.26 \pm 1.14$ \\
17 & The Bourne Identity & sadness & $1.52 \pm 0.59$ & $3.00 \pm 0.74$ & $3.96 \pm 0.77$ \\
18 & The Shawshank Redemption & surprise & $2.65 \pm 0.78$ & $3.91 \pm 0.85$ & $3.57 \pm 1.04$ \\
\hline \hline
\end{tabular}

Fig. 5. DREAMER database films and expected emotions

The length of the film clips was between 65 to $393 \mathrm{~s}(\mathrm{M}=199 \mathrm{~s})$, which is considered sufficient since, according to psychologists, video stimuli between 1 to $10 \mathrm{~min}$ is capable of detecting single emotions [20]. The target emotions after the experiment were Valence, Arousal, or Dominance. The database has results based on levels of subjects after watching the corresponding films. The Scale is between 1 (lowest) to 5 (highest) reactions for each Valence, Arousal, and Dominance measurements. The database provides information about Affect Recognition therefore it was possible to use it in further Machine Learning experiments. The dataset of film clips: 1, 2, 4, 5, 6, 7, 9, 12, 14 were chosen.

The dimensional approach proposes that every emotion has different amounts of hedonic and arousal value. In the DREAMER database, there are three different emotions based on different film clips. This means in the Database, there are measurements of levels for each emotion after different film clips.

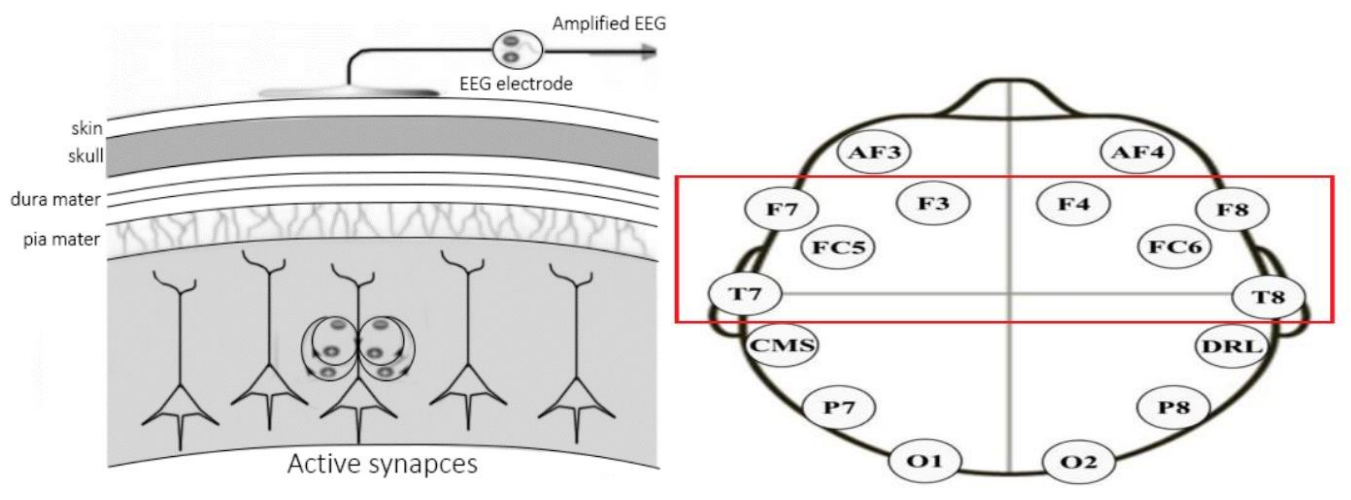

Fig. 6. The source of EEG signals (left) and tThe corresponding EEG nodes in prepared Dataset

\subsection{Valence, Arousal and Dominance}

Wundt (1912/1924) was among the first psychologists to argue that affective experiences involve at least two properties: valence (ranging from feeling pleasant to unpleasant) and arousal (ranging from feeling quiet to active) [24]. In Psychology, emotional valence describes the measurement to which an emotion is negative or positive, and arousal refers to its intensity. Dominance on the other hand represents the control and influence over circumstances [25]. It is explained in the section on Emotions and Neurotransmitters and it is shown in figure 6. The emotions have levels as explained in Dimensional Theory of Emotion [11], therefore approach in DREAMER Database using 1 to 5 to determine the levels of each emotion suits the experiment.

\subsection{Algorithms}

In this project, SVM, Logistic Regression, KNN, XGBoost, and Random forest (RF) were used. RF is a bagging technique and XGBoost is a boosting technique. RF gave the highest accuracy. The algorithm takes votes of the similar or same implementations, XGBoost on the other hand, improves the weights of the model [23]. Each model, in this case, is influenced by the previous implementation. Neural Networks may give acceptable results when the Data-set is big enough. A Dense Neural Network means (DNN) all the nodes in each layer are connected. Before a neural network can be used for the required problem or task, it must be trained. 
The learning is based on given rules and data. After determined (100 epochs and 500 batch size and 0.01 learning rate in this project) epochs, the NN weights the connections of the neurons afterward, it develops patterns, relations which also means intelligence. The learning rules depend on how the learning material changes the neural network iteratively. Thus the Neural Network learns how to connect the weights and relations according to its neurons properly.

The RELU (Rectified Linear Units) activation function is used in input and hidden layers. The advantages of using RELU are: it converges faster than traditional activation functions (tan-h, sigmoid), and more particularly; RELU is nonsaturation of its gradient, which means it accelerates the convergence of stochastic gradient descent [21]. Therefore RELU is used as the main activation function of neurons. The Soft-max gives at least a minimal amount of probability to all elements in the output vector [22]. The Soft-Max activation function was used for multi-class classification. Data was scaled using Min-Max before the training. Neural network modelling requires knowledge of historical data on supply volume large enough as to provide a good training of the network [29].

The shape of the neural network might be related to the number of features, in this case; the training data has nine features( 8 EEG columns and age column) for the model evaluation and training, NN has 9 input neurons, two hidden layers with 18 hidden layer units (nodes), and 9 output units shown in the figure below.

\begin{tabular}{|c|c|c|}
\hline $\begin{array}{l}\text { Layer (type) } \\
============== \\
\text { dense (Dense) }\end{array}$ & $\begin{array}{l}\text { Output Shape } \\
============ \\
\text { (None, 9) }\end{array}$ & $\begin{array}{l}\text { Param \# } \\
========== \\
90\end{array}$ \\
\hline dense_1 (Dense) & (None, 18) & 180 \\
\hline dense_2 (Dense) & (None, 18) & 342 \\
\hline dense_3 (Dense) & (None, 9) & 171 \\
\hline
\end{tabular}

Fig. 7. Structure of Neural Network visualization in python

\subsection{K-Means Algorithm}

K-Means is an Unsupervised Learning algorithm. Unsupervised algorithms make inferences from datasets using only input vectors without referring to known, or labelled, outcomes. This means for the K-Means algorithm the synthetic Data (generated using GAN) and original Data were used without labels.

\subsection{Generative Adversarial Networks}

A Generative Model is an unsupervised approach in ML that automatically discovers and learns the patterns in input data therefore the model can be used to generate or new data that could have been drawn from the original dataset [26]. It is possible to use GAN to generate Tabular data [27], in this research Conditional GAN for a Tabular data model was used (CTGAN). CTGAN outperforms all methods to date and surpasses Bayesian networks on at least $87.5 \%$ of tested datasets [27]. And conditional generator in GTGAN and training-by-sampling can overcome the imbalanced training data issue [27]. DREAMER database is a big and not balanced tabular database therefore this method was chosen.

\begin{tabular}{|c|c|c|c|c|c|c|c|c|c|c|}
\hline & F7 & $\mathbf{F 3}$ & FC5 & $T 7$ & T8 & FC6 & F4 & F8 & Participant & Age \\
\hline 0 & 4203.552046 & 4156.127160 & 4382.279428 & 3864.216931 & 4279.113371 & 3833.542173 & 4463.083137 & 4311.492870 & 7 & 24 \\
\hline 1 & 4307.395474 & 4410.086663 & 4412.589617 & 4060.456142 & 4000.183823 & 4073.934100 & 4498.636255 & 4269.469152 & 17 & 24 \\
\hline 2 & 3995.793459 & 4169.346434 & 4401.219550 & 4286.014600 & 4256.597210 & 3984.843198 & 3930.496456 & 4303.901104 & 17 & 23 \\
\hline 3 & 4090.673105 & 4386.967971 & 4179.603535 & 4083.889777 & 4380.213973 & 3906.740936 & 4095.513307 & 4584.518766 & 2 & 28 \\
\hline 4 & 4097.154870 & 4168.952474 & 4116.434074 & 4336.664542 & 4381.233043 & 4537.257813 & 3960.494053 & 4499.348790 & 1 & 23 \\
\hline
\end{tabular}

Fig. 8. Synthetic Data (GAN) in tabular format is shown

\section{Results}

\subsection{Random Forest Algorithm using Original Dataset}

The first method was RF (supervised learning) for multi-class classification. After training and testing, (70\% train, $30 \%$ test data) models were able to predict with $98 \%$ accuracy. There were five different classes for three of these groups in the Dataset. The classification groups are individual which means they do not affect each other. One more important issue here was not all the classification groups have five different levels. In this case, Arousal for film 4 had only 3 , 4, and 5 while the Valence group has 1,2,3,4, and 5 as classes. RF tests were completed for 9 different films as explained in the previous chapter and accuracies were significantly close to each other around $\% 98$ for both female and male groups. 


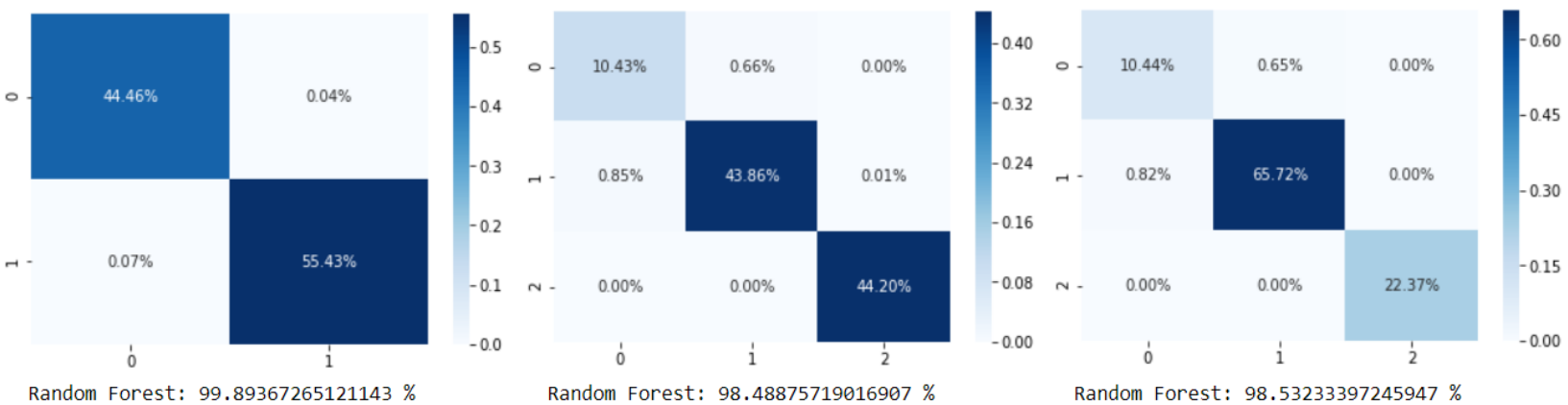

Fig. 9. Random Forest results of arousal, dominance and valence shown for Film 4

\subsection{Dense Neural Network Using Original Dataset}

DNN (Dense Neural Network) was used and tested for 9 films. Data was shuffled and used the same Random state (42) and cross-validation techniques to prevent overfitting. The accuracy for DNN was $91.41 \%$ for male candidates for film 4 to classify Dominance. The accuracy was 92.44 for female candidates for film 4 to classify Dominance. After testing Valence and Arousal the results were similar between 90\% - 95\% accuracy for males and females. There were no overfitting (Train-Val was close to each other) during the training process. Therefore the model gave high accuracy to detect emotion levels (1 to 5) as a multi-class classification for Valence, Arousal, and Dominance Separately.
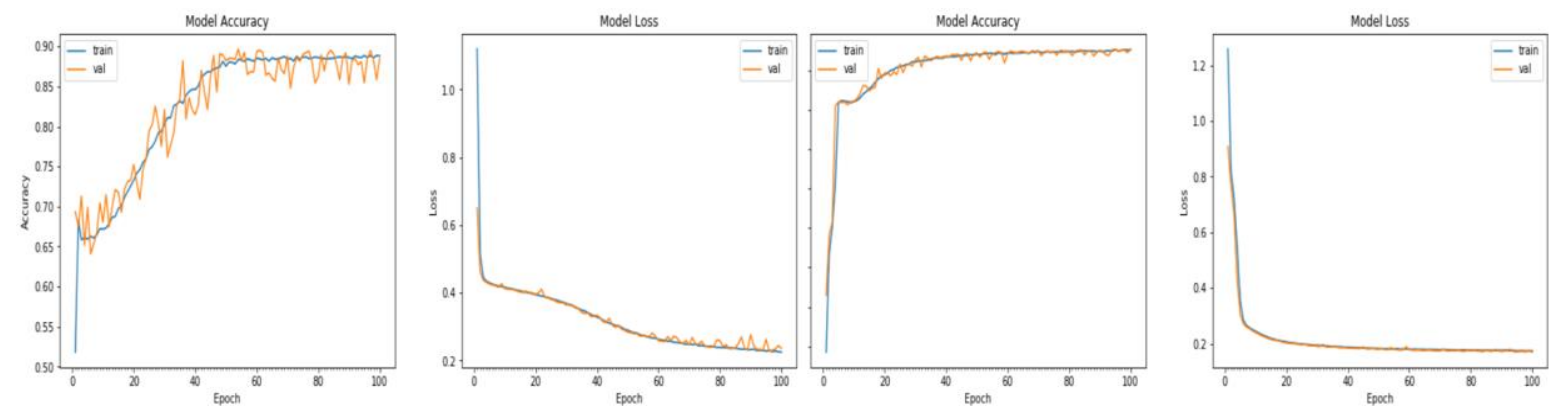

Fig. 10. DNN implementation results for dominance, male (left) and for female (right)

\subsection{K-Means using Original Dataset}

The unsupervised learning method K-Means was applied to the original Dataset. For film14 Dataset. The results of dataset film14-Females were clustered properly on the other hand the results of dataset film14-Male were not reliable shown in the figures below. The purpose of this test was to detect the possible clusters related to Dominance, Valence, or Arousal using an unsupervised learning approach. Elbow method using the edge on graph was used that selects the optimal number of clusters by fitting the model with a range of values for $\mathrm{K}$.
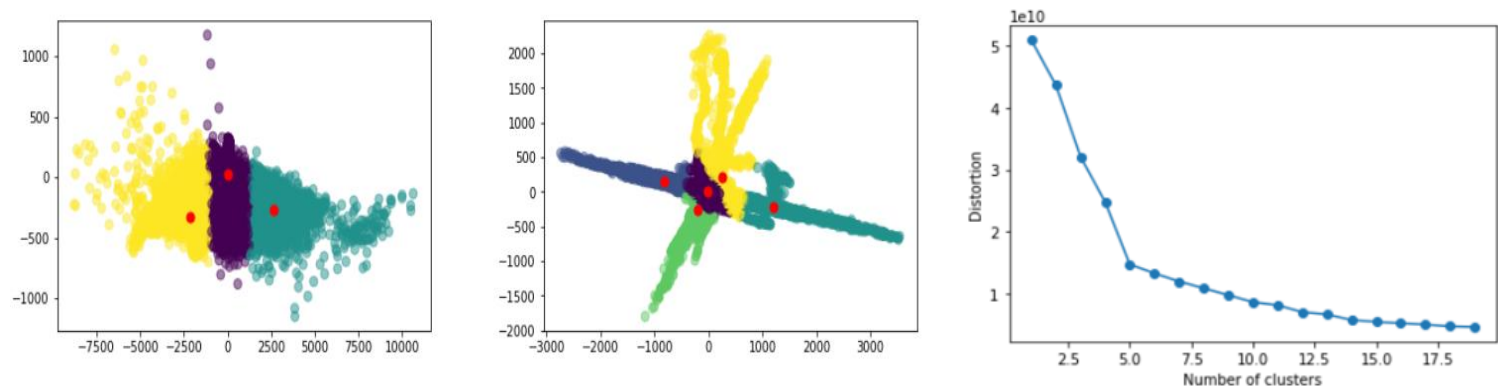

Fig. 11. K-Means and Elbow results for film14 Female (left) and Male (Right) respectively.

\subsection{Generative Adversarial Networks for Synthetic Data Generation}

To create Tabular Data using GAN there were 300 epochs for generator and discriminator. To observe the effect of quantity of GAN data; three different sample numbers of Data as 1000, 10000, and the original number of subjects, for instance, there were 25472 samples for females in film-1 or 21760 for females in the film- 12 dataset. 
The correlation results between original and Synthetic data are shown in the figure below. To test the generated data, the same supervised (RF and DNN) and unsupervised (K-Means) algorithms were used.
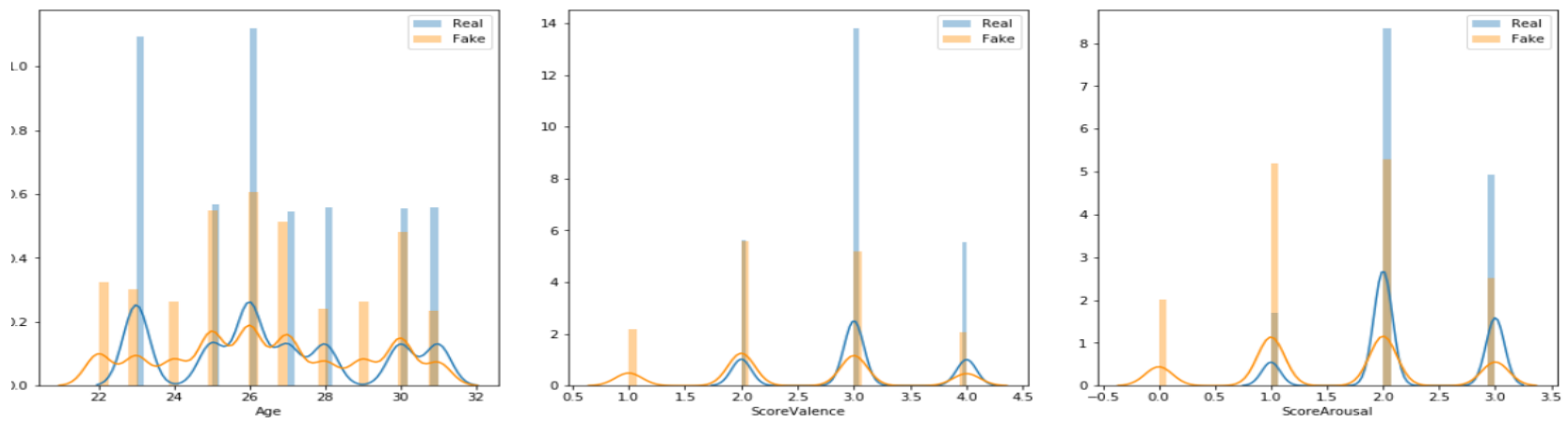

Fig. 12. GAN results of attributes(correlations) for Synthetic Data generation

\subsection{Random Forest and DNN using Synthetic Data}

Synthetic Data (GAN) was tested for all 9 films using 1000, 10000, and original dataset size (chosen). Dataset size did not affect accuracy. The best results were Female Film1 Dominance 51.67\%, Female Film14 Valence 51.3\%, Male Film12 Valence 42\%, Male-Film14 Dominance 41.67\%. Arousal gave the worst results around 30\% in every dataset. An extra algorithm SVM (Support Vector Machine classifier) was used and it slightly performed better than RF in FemaleFilm1 Dominance. The highest accuracies were in films 1, 12, and 14, Dominance and arousal data for both genders and synthetic data of Female subjects performed better up to $10 \%$ accuracy difference.

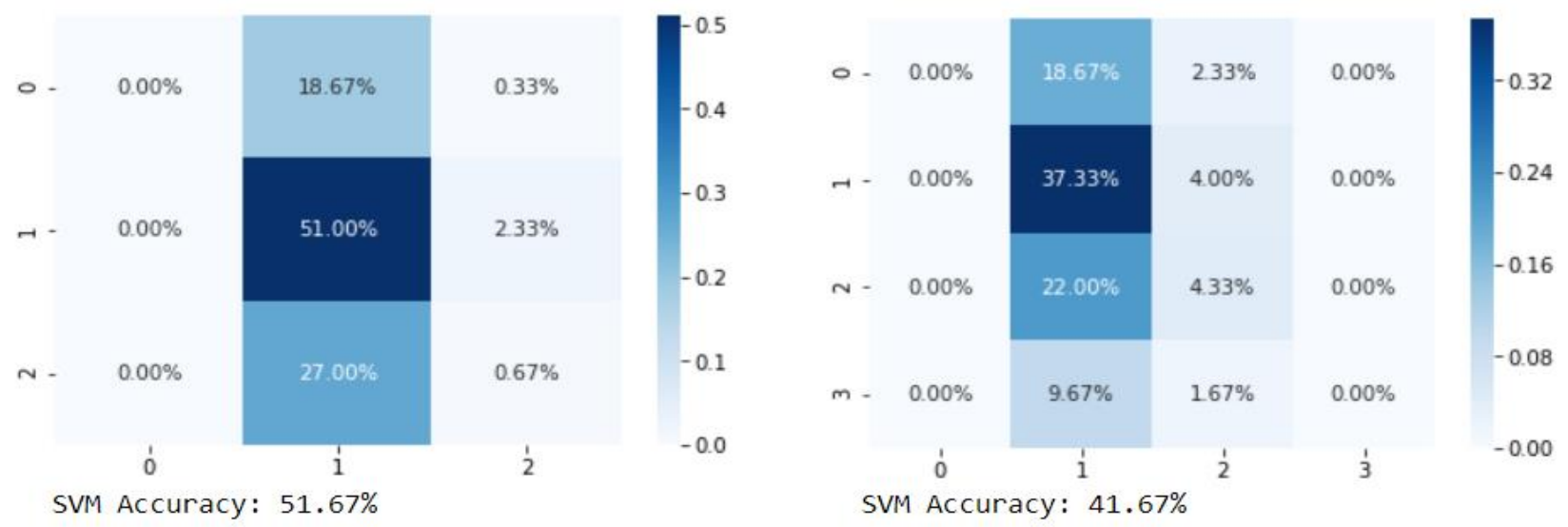

Fig. 13. Dominance -Film1 Female and film 14 Male Supervised learning results for Synthetic Dataset

\subsection{K-Means Using Synthetic Data}

The same approach above was implemented using synthetic (GAN) data. The results were similar to the original data tests. In each unsupervised test, algorithms have failed to determine the exact numbers of clusters although the optimal number of clusters was 5 according to the Elbow Method. The results of K-Means were inconsistent.
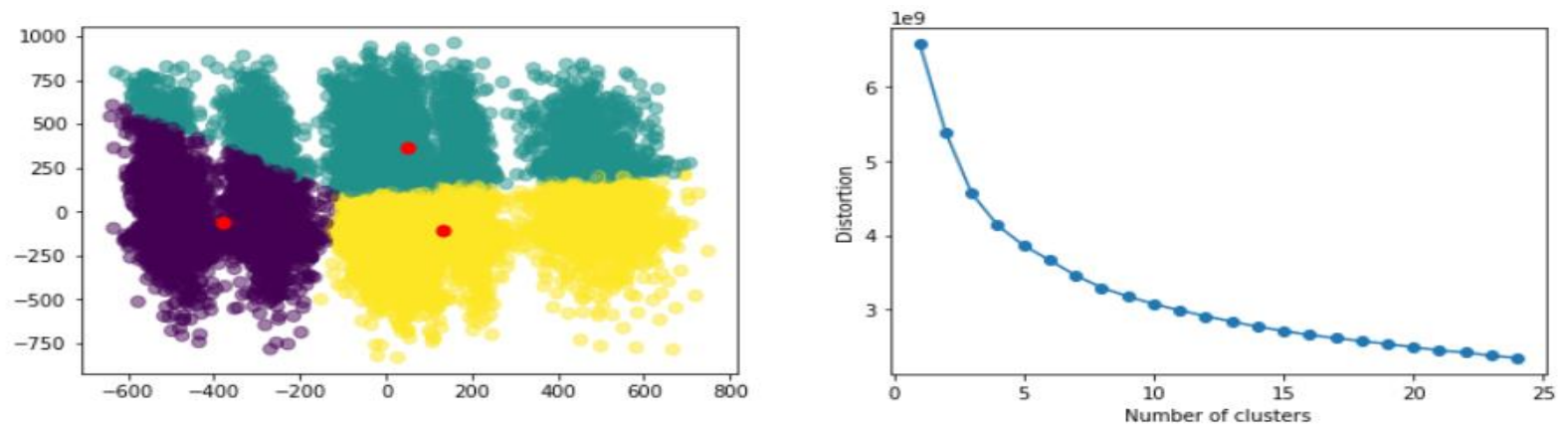

Fig. 14. Results of K-Means and Elbow Method implementation using synthetic data 


\section{Discussion}

In this paper, the structure of the brain, limbic system, neurotransmitters, and emotion theories were explained. Based on two different emotional theories, it is possible to say that emotions, limbic system work together with other parts of the brain such as decision making and memory. Grossberg and Levine's theory proposed that emotions are the neural signals that connect the instinctual sensor with the conscious brain, to make the instinctual needs to be consciously known to the brain, to make the instinct conceptually recognized-understood [12]. The basic emotions are evoked by sensing the basic bodily instinctual needs, while the "complex" emotions, including love, aesthetic emotions are evoked by higher cognitive needs of a human being [13]. In Maslow's Hierarchy of Needs, physiological needs and safety needs might be directly related to basic emotions, while the other needs, such as the need for love, esteem, and self-actualization, are related to feelings such as aesthetic emotions [13].

There are two different generalizations for emotions. Firstly basic emotions are related to our instincts. The second one is emotions by experience that are complex to detect and analyse. Experience learning is related to personal behaviour, this means a subject might have arousal or valence based on her desires, wishes, or experiences. Thus to analyse complex emotions using this database was not possible. The tests were related to the dimensional theory of emotion using basic emotions as valence, arousal, and dominance in the DREAMER dataset. The research was able to classify rankings of emotions although it was not possible to specify which exact emotion is occurring in the brain during the DREAMER data experiments. The neurotransmitter dopamine (DA), is known to mediate unconditioned pleasure and reward from food, sex, and drugs, but recent findings suggest that DA is also involved in the anticipatory, preparatory, approach, and coping phases of reward behaviour [14]. Alterations in one neurotransmitter affect other neurotransmitters. Numerous studies have pointed to an important role for neuromodulators (e.g., DA, 5-HT, and NE) in the emotional process [15]. There are relationships between higher levels of arousal and higher relation of dopamine [16] [17]. Based on this research, we can say that neurotransmitters control our emotions and EEG might detect signal alterations in the human brain. Thus using EEG signals, it was possible to analyse rankings of basic emotions.

The supervised learning classifications for original data were successful with $95-98 \%$ accuracy. Thus, it is possible to say that using EEG data rankings of basic emotions can be classified. On the other hand, unsupervised learning tests were inconsistent to create clusters analysing EEG data. GAN has been tested to generate synthetic EEG data to widen the experiments on the database. The highest accuracy was $51 \%$ on the female subject using GAN data for supervised learning algorithms. GAN results were not high enough as in the original dataset although it is possible to say that GAN was able to generate synthetic EEG data with around $50 \%$ accuracy.

\section{Conclusion}

In conclusion, emotions in the human brain were analysed. The limbic system not only controls emotions but also works with other parts of the brain. Neurons are the tiniest units in the brain that are synchronously communicating together. Neurotransmitters allow the communication process electrochemically. Because of the relation between emotions, neurotransmitters, and EEG signals, it is possible to detect alterations in neurotransmitter levels and using those signals occurring in the brain to perform Affect Recognition. The multi-output multi-class accuracies were high enough to use the model in real-time equipment. In this research some of the results were acceptable. Using different methods and models, not all models gave high accuracies and there were unstable results for several subjects. Emotions are still unknown in theory and practice and this issue was the biggest limitation of this research because different subjects can give different results for ML systems which will cause unstable models. Not only neurotransmitters but experience, memory, and expectations can affect emotions. It is not yet possible to generalize emotions based on experiments. The goal is to help patients in hospitals by understanding basic emotions. Unfortunately, using such systems might have dangerous outcomes related to privacy. For future work, the Machine Learning model will be tested using Cyber-Physical and control systems. Finally, the EEG signals can be used to develop an Affect Recognition system to analyse or detect basic emotions. Therefore human-computer interface can be used in the healthcare industry to help patients in hospitals or to improve the quality.

\section{Acknowledgments}

Firstly, I would like to thank my father Dr. Turgay Ünal for his help in EEG studies and would like to thank Associate professor Vyacheslav Potekhin for all his help during my studies. Finally, I thank my mom Dr. Seher Gülçin Ünal for her assistance in giving all the support in my life. This research is being conducted at the Peter the Great St. Petersburg Polytechnic University and partially funded by the Ministry of Science and Higher Education of the Russian Federation as part of World-class Research Center programme: Advanced Digital Technologies (contract No. 075-15-2020-934 dated 17.11.2020).

\section{References}

[1] Marieb, H. \& Hoehn, K. (2009). Brain Anatomy Adapted from Human Anatomy \& Physiology 9th edition, Pearson, pp. 335, ISBN-13: 9780321743268 
[2] Brisch, R.; Saniotis, A. \& Rainer, W. (2014). The role of dopamine in schizophrenia from neurobiological and evolutionary perspective: old fashioned, but still in vogue, Frontiers in Psychiatry, Volume 5, pp. 1-12 doi: 10.3389/fpsyt.2014.00047

[3] John, A. \& Putman, A. (2012). Signal Processing Techniques, EEG Info, USA, pp. 3-15, doi:1s10.1061/ (ASCE) CP.1943-5487.0000719

[4] Asli, A. (2015). EEG Elektroensephalogram, Bio-physics YDU Medicine Faculty Biophysics, pp.3-11, doi: 10.5350/BTDMJB201107303

[5] Bright Brain Centre. (2021).zEEG- a Window into the Real Time Neural Processing, Available from: cwww.brightbraincentre.co.uk/electroencephalogram-eeg-brainwaves Accessed: 2021-07-23

[6] Marianne K.; Daniella S.; Ariel R.; David N.; Jackson, C. \& Ricardo xG. (2019). Dopamine: Functions, Signaling, and Association with Neurological Diseases, Cellular and Molecular Neurobiology, pp.1-30, doi: 10.1007/s10571$\mathrm{x}$ x018-0632-3

[7] Engin U. (2015). The Impact of Neuroanatomical and Neurochemical Structure of Brain on Personality and Behaviour, International Journal of Alanya Faculty of Business, ResearchGate, pp. 193-219

[8] Potekhin, V.; Muhammed, E. \& Ogul, U. (2018). Development of Artificial Neural Network To Determine Hand Activity Using Real Motor Activity From Electroencephalography Signals, Apple Space Engineering, Technologies and Exploration, pp.214-224., ISBN 978-3-00-060580-2

[9] Zeljko, T.; Cosic, I. \& Katalinic B. (2019). Manufacturing And The Rise Of Artificial Intelligence, Innovation Challenges Innovation Challenges, Proceedings of the 30th DAAAM International Symposium, pp. 192x196.Published by DAAAM International, ISBN 978-3-902734-22-8, doi:10.2507/30th.daaam.proceedings.025

[10] Fushun, W.; Yang, J.; Fang, P.; Roger, C. \& Jason H. H. (2020). Neurotransmitters and Emotions, Frontiers in Psychology Emotion Science, pp. 1-5, doi: 10.3389/fpsyg.2020.00021

[11] Simeng, G.; Fushun, W.; Nitesh, P.; Patel, J. \& Jason H. H. (2019). A Model for Basic Emotions Using Observations of Behavior in Drosophila, Frontiers in Psychology Emotion Science, pp. 1-10, doi: 10.3389/fpsyg.2019.00781

[12] Yi, L.; Zhao, J. \& Wenbin, G. (2018). Emotional Roles of Mono-Aminergic Neurotransmitters in Major Depressive Disorder and Anxiety Disorders, Frontiers in Psychology Emotion Science, pp.x1-9, doi: 10.3389/fpsyg.2018.02201

[13] Zhang, K.; Guo, J.; Peng, Y.; Xi, W. \& Guo A. (2007). Dopamine-mushroom body circuit regulates saliency-based decision-making in Drosophila. Science 316, pp. 1901-1904, doi: 10.1126/science.1137357

[14] Schultz, W.; Dayan, P. \& Montague, P. R. (1997). A neural substrate of prediction. Science 275,pp. 1593-1599, doi: 10.1126/science. 275.5306 .1593

[15] Pereira, T. \& Murthy, M. (2017). To fight or not to fight. Neuron 95, pp.986-988, doi: 10.1016/j.neuron.2017.08.029

[16] David, C.; Vandana P.; Deborah, S. \& Don, B. (1987) The relationship of affiliative arousal to dopamine release, Springer-Motivation and Emotion volume 11, pp. 51-66, doi: 10.1007/BF00992213

[17] Jan, W. (2018). Effects of positive emotion, extraversion, and dopamine on cognitive stability-flexibility and frontal EEG asymmetry, Pubmed Psychophysiology, pp. 1-10, doi: 10.1111/psyp.12727

[18] Solomon D. (2020). nervous system. Encyclopedia Britannica, Available from: www.britannica.com/science/nervous-system Accessed: 2021-08-13

[19] Gabert-Quillen, C.; Bartolini, E. \& Abravanel, B. (2015). Ratings for emotion film clips, Behavior Research Methods, vol. 47, no. 3, pp. 773-787, doi: 10.3758/s13428-014-0500-0

[20] Katsigiannis, S. \& Ramzan, N. (2017). DREAMER: A Database for Emotion Recognition Through EEG and ECG Signals from Wireless Low-cost Off-the-Shelf Devices, IEEE Journal of Biomedical and Health Informatics, pp.110, doi: 10.1109/JBHI.2017.2688239

[21] Krizhevsky, A.; Ilya, S. \& Geoffrey H. (2012). ImageNet Classification with Deep Convolutional Neural Networks, Advances in neural information processing systems, pp. 1-9., ISSN 1049-5258, doi: 10.1145/3065386

[22] Nielsen, M. (2019). Neural Networks and Deep Learning, Determination Press, Unpublished

[23] Blockeel, H. \& Raedt, L. (1997). Top-down induction of first-order logical decision, Information Technologies, pp. 285-297, doi: 10.1007/978-3-662-04599-2_6

[24] Kuppens, P.; Tuerlinck F.; Russell, J. \& Feldman B. (2012). The Relation Between Valence and Arousal in Subjective Experience, Psychological Bulletin 139, pp.1-10, doi: 10.1037/a0030811

[25] Sheri, L.; Liane, J.; \& Luma, M. (2012). The Dominance Behavioral System and Psychopathology: Evidence from Self-Report, Observational, and Biological Studies, NCBI, pp. 1-12, doi: 10.1037/a0027503

[26] Ian, J.; Jean, P; Mehdi, M.; Bing, X.; Warde, D.; Ozairy, S.; Courville, A. \& Bengio, Y. (2014). Generative Adversarial Nets, Montreal University, pp. 1-9

[27] Lei, X. \& Kalyan, V.(2018). Synthesizing Tabular Data using Generative Adversarial Networks, MIT, pp. 1-12

[28] Katalinić, B.; Kostenko, D.; Onufriev, V. \& Potekhin, V. (2020). Cyber-Physical Systems in Complex Technologies and Process Control, pp. 40-54., Lecture Notes in Networks and Systems, doi: 10.1007/978-3-030-34983-7-5

[29] Ciupan, E. (2013). A Study Regarding the Possibility of Optimizing the Supply Batch using Artificial Neural Networks, 24th DAAAM International Symposium on Intelligent Manufacturing and Automation, pp. 141-149, doi: 10.1016/j.proeng.2014.02.214 